\title{
ON EXPONENTIAL BASES AND FRAMES WITH NON-LINEAR PHASE FUNCTIONS AND SOME APPLICATIONS
}

\author{
JEAN-PIERRE GABARDO, CHUN-KIT LAI, AND VIGNON OUSSA
}

\begin{abstract}
In this paper, we study the spectrality and frame-spectrality of exponential systems of the type $E(\Lambda, \varphi)=\left\{e^{2 \pi i \lambda \cdot \varphi(x)}: \lambda \in \Lambda\right\}$ where the phase function $\varphi$ is a Borel measurable which is not necessarily linear. A complete characterization of pairs $(\Lambda, \varphi)$ for which $E(\Lambda, \varphi)$ is an orthogonal basis or a frame for $L^{2}(\mu)$ is obtained. In particular, we show that the middle-third Cantor measures and the unit disc, each admits an orthogonal basis with a certain non-linear phase. Under a natural regularity condition on the phase functions, when $\mu$ is the Lebesgue measure on $[0,1]$ and $\Lambda=\mathbb{Z}$, we show that only the standard phase functions $\varphi(x)= \pm x$ are the only possible functions that give rise to orthonormal bases. Surprisingly, however we prove that there exist a greater degree of flexibility, even for continuously differentiable phase functions in higher dimensions. For instance, we were able to describe a large class of functions $\varphi$ defined on $\mathbb{R}^{d}$ such that the system $E(\Lambda, \varphi)$ is an orthonormal basis for $L^{2}[0,1]^{d}$ when $d \geq 2$. Moreover, we discuss how our results apply to the discretization problem of unitary representations of locally compact groups for the construction of orthonormal bases. Finally, we conclude the paper by stating several open problems.
\end{abstract}

\section{INTRODUCTION}

1.1. Definition and Background. Let $\Lambda$ be a countable set in $\mathbb{R}^{d}$. We denote by $E(\Lambda):=\left\{e^{2 \pi i \lambda \cdot x}: \lambda \in \Lambda\right\}$ the collection of exponential functions with frequencies in $\Lambda$. For a fixed positive Borel measure $\mu$ defined on a subset $\Omega$ of $\mathbb{R}^{d}$, perhaps one of the most profound and largely unresolved questions in harmonic analysis is to characterize pairs of the type $(\Omega, \Lambda)$ such that $E(\Lambda)$ is either an orthogonal basis or a Riesz basis or a frame for $L^{2}(\Omega, d \mu)$. Although the literature contains several significant results, which we summarized below, at this point, this problem remains unresolved even in a variety of concrete cases.

First, we recall that a finite Borel measure is a spectral measure if there exists a countable discrete set $\Lambda$ called the spectrum, such that $E(\Lambda)$ forms an orthogonal basis for $L^{2}(\mu)$. Additionally, if there exists a countable set $\Lambda$ such that $E(\Lambda)$ forms an orthogonal basis for $L^{2}(\Omega)$, we say that $\Omega$ is a spectral set (equivalently, the measure $\chi_{\Omega} d x$ is a spectral measure). The problem of characterizing spectral sets was initiated by Fuglede [9], who conjectured in 1974 that a set is spectral if and only if one can tile the whole Euclidean space $\mathbb{R}^{d}$ using translates of that set. Although Fuglede's conjecture in its full generality was disproved by Tao [25] in 2004, the problem remains open in many special settings. As a partial solution to this conjecture, Lev and Matolcsi recently settled affirmatively the case where $\Omega$ is a convex set [20]. Moreover, Jorgensen and Pedersen [16] were the first to discover spectral measures that are singularly continuous with respect to the Lebesgue meaure. They found that the middle-fourth Cantor measure is a spectral measure, while

2010 Mathematics Subject Classification. 42C15.

Key words and phrases. Expoential bases, frames, and non-linear phase functions. 
the middle-third Cantor measure is not. The reader interested in learning more about fractal spectral measures can consult the following survey [6] authored by Dutkay, Lai, and Wang.

Next, let us mention the notion of frame, which generalizes the notion of basis, in a separable Hilbert space $\mathcal{H}$ and were first introduced by Duffin and Schaeffer [4] in order to deal with nonharmonic Fourier series. Given a countable index set $\mathcal{N}$, a collection of vectors $\left\{x_{n}\right\}_{n \in \mathcal{N}}$ in $\mathcal{H}$ is called a frame if there exist positive constants $A, B$ such that

$$
A\|x\|^{2} \leq \sum_{n \in \mathcal{N}}\left|\left\langle x, x_{n}\right\rangle\right|^{2} \leq B\|x\|^{2}, \quad x \in \mathcal{H} .
$$

In that case, every element $x$ of $\mathcal{H}$ admits an unconditional expansion $x=\sum_{n \in \mathcal{N}} c_{n} x_{n}$, in terms of the frame elements $x_{n}$, for certain coefficients $c_{n}$. In the particular case where $\mathcal{H}=L^{2}(\mu)$ and $\left\{x_{n}\right\}_{n \in \mathcal{N}}=E(\Lambda)$ (where each exponential in $E(\Lambda)$ is viewed as an element of $L^{2}(\mu)$ in the obvious way), we call $E(\Lambda)$ a Fourier frame for $L^{2}(\mu)$ if the inequalities above hold. Thus, more explicitly, this means that there exist constants $0<A \leq B<\infty$ such that

$$
A\|f\|_{L^{2}(\mu)}^{2} \leq \sum_{\lambda \in \Lambda}\left|\int f(x) e^{-2 \pi i \lambda \cdot x} d \mu(x)\right|^{2} \leq B\|f\|_{L^{2}(\mu)}^{2}, \forall f \in L^{2}(\mu) .
$$

When such a frame exists, we call $\mu$ a frame-spectral measure and $\Lambda$ a frame-spectrum. Fourier frames provide thus, for each $f \in L^{2}(\mu)$, a basis-like expansion of the type

$$
f=\sum_{\lambda \in \Lambda} c_{\lambda} e^{2 \pi i \lambda \cdot x}
$$

for some (possibly non-unique) square-summable sequence $\left(c_{\lambda}\right)_{\lambda \in \Lambda}$. The redundancy property built into the structure of frames makes the expansion of vectors described above robust to the loss of data, and as such, frames are very useful in signal transmission. In cases where the coefficients used to represent vectors are unique, the system $E(\Lambda)$ is called a Riesz basis and $\mu$ a Riesz-spectral measures. We refer readers interested in a detailed treatment of the theory of frames and Riesz bases to the monographs of Heil and Christensen [2, 14].

Regarding the existence of exponential frames and Riesz bases, there is a general interest in determining measures that are Riesz-spectral and frame-spectral. A theory addressing these issues was established in [13, and the existence of exponential Riesz basis on a finite union of rectangles on $\mathbb{R}^{d}$ was recently confirmed by Kozma and Nitzan [18, 19]. It is worth noting that despite the intense efforts devoted to this line of research, to this date, several concrete cases are still far from being settled. For instance, the following questions have yet to be resolved (see [24, 6])

(i) Does the middle-third Cantor measure admit a Fourier frame?

(ii) Do a triangle or a disk admit an exponential Riesz basis?

1.2. Exponential functions with non-linear phases. Our main goal here is to consider a different question regarding frames generated by measures, which we will always assumed to be bounded.

Let $\mu$ be a finite Borel measure with closed support $K_{\mu}$. Let $\varphi$ be a Borel measurable function defined on a set containing $K_{\mu}$ and $\Lambda$ be a countable and discrete set. We define 
the collection of generalized exponentials with phase functions $\varphi$ as

$$
E(\Lambda, \varphi)=\left\{e^{2 \pi i \lambda \cdot \varphi(x)}: \lambda \in \Lambda\right\}
$$

We aim to address the following question:

Question 1 . Let $\mu$ be a finite Borel measure on $\mathbb{R}^{d}$ with support $K_{\mu}$. Can we characterize the pairs $(\Lambda, \varphi)$ for which the system $E(\Lambda, \varphi)$ is an orthonormal basis, or a frame for $L^{2}(\mu) ?$

Note that $E(\Lambda, \varphi)$ forms a frame if and only if (1.1) holds when the exponential function $e^{2 \pi i \lambda \cdot x}$ is replaced with $e^{2 \pi i \lambda \cdot \varphi(x)}$. The motivation of the work is manifold. On the one hand, we are are interested in the flexibility afforded by $\varphi$ in forming exponential bases/frames for $L^{2}(\mu)$. On the other hand, complex exponential systems with non-linear phases arise naturally in the realization of unitary representations of non-commutative Lie groups. Such systems have been investigated from a representation-theoretic viewpoint in [12, 22, 23, 21. The overarching theme in these projects is concerned with the question of discretizing a unitary (irreducible) representation of a locally compact group for the construction of frames and orthonormal bases. These questions are deeply connected to wavelet theory, and time-frequency analysis [1, 2, 11] and have been studied with varying degrees of generality [11, 12, 22, 21, 7].

1.3. Main Results and organization. This paper aims to present a mathematical framework on the spectrality and frame-spectrality of $E(\Lambda, \varphi)$ for a general finite Borel measure $\mu$. Our main result gives a complete characterization of pairs $(\Lambda, \varphi)$ for which the system of vectors $E(\Lambda, \varphi)$ forms a frame or a basis for $L^{2}(\mu)$. The work is organized as follows.

In the second section, we define the concept of essential injective Borel measurable functions. This notion plays a central role in the proof of our main result, which is stated in Theorem 3.2.

In Section 4, we will illustrate our main result with some specific examples. In particular, we show that the middle-third Cantor measure admits an orthonormal basis of the form $E(\Lambda, \varphi)$ for some Borel-measurable function $\varphi$. Additionally, we also establish that the unit disc also admits an orthonormal basis of the type $(\Lambda, \varphi)$ where $\varphi$ is a piecewise continuously differentiable phase function. These examples clearly illustrate how allowing the presence of $\varphi$ in the "generalized" exponentials we consider greatly simplify the problem of constructing orthonormal bases or frames in this setting.

In Section 5, we consider the problem of determining which continuous function $\varphi$ have the property that the system $E(\mathbb{Z}, \varphi)$ is an orthonormal basis for $L^{2}[0,1]$. We show that if $\varphi$ preserves measure zero sets, the only such $\varphi$ are exactly $\varphi(x)= \pm x$.

In Section 6, we show that, in the multidimensional setting, we cannot expect results analogous to those obtained in the one-dimensional case. We also describe in Theorem 6.1 a fairly large class of functions $\varphi$ such that $E\left(\mathbb{Z}^{d}, \varphi\right)$ is an orthonormal basis for $L^{2}[0,1]^{d}$. When $d=2$, we present sufficient conditions under which we cannot improve the construction given in Theorem 6.1.

In Section 7, we explore applications to the discretization of unitary representations of a class of Lie groups for the construction of orthonormal bases [11, 12, 22, 23, 21, 7].

Finally, in Section 8, we conclude our paper by stating several open questions. 


\section{EsSENTIAL INJECTIVITY}

In this section, we define and discuss the property of essential injectivity of a Borel map. Let $\mu$ be a finite Borel measure on $\mathbb{R}^{d}$ with support $K_{\mu}$. Given a Borel measurable map $\varphi: K_{\mu} \rightarrow \mathbb{R}^{d}$, the pushforward measure of $\mu$ under $\varphi$ is characterized by the property

$$
\varphi_{*} \mu(E)=\mu\left(\varphi^{-1}(E)\right), \quad E \subset \mathbb{R}^{d}, \quad E \text { Borel. }
$$

Moreover, integration with respect to the measure $\varphi_{*} \mu$ satisfies the following formula.

$$
\int f(y) d\left(\varphi_{*} \mu\right)(y)=\int f(\varphi(x)) d \mu(x), \quad f \in C_{0}\left(\mathbb{R}^{d}\right),
$$

where $C_{0}\left(\mathbb{R}^{d}\right)$ denotes the space of continuous functions on $\mathbb{R}^{d}$ that vanish at infinity. The concept of essential injectivity as defined below plays a central role in this work.

Definition 2.1. Given a finite Borel measure $\mu$ with support $K_{\mu}$, a Borel measurable function $\varphi: K_{\mu} \rightarrow \mathbb{R}^{d}$ is said to be $\mu$-essentially injective if for any $f \in L^{2}(\mu)$, there exists $h \in L^{2}\left(\varphi_{*} \mu\right)$ such that $f(x)=h(\varphi(x)) \mu$-a.e.

We will record some conditions equivalent to essential injectivity in the following lemma.

Lemma 2.2. Let $\varphi: K_{\mu} \rightarrow \mathbb{R}^{d}$ be Borel measurable and let $\nu=\varphi_{*} \mu$ be the corresponding pushforward measure. Then, the following are equivalent.

(1) The span of the collection $\chi_{\varphi^{-1}(F)}, F \subset \mathbb{R}^{d}, F$ Borel, is dense in $L^{2}(\mu)$.

(2) For any $f \in L^{2}(\mu)$, there exists $h \in L^{2}(\nu)$ such that $f(x)=h(\varphi(x)) \mu$-a.e.

(3) There exists a Borel set $\mathcal{N} \subset K_{\mu}$ with $\mu(\mathcal{N})=0$ such that $\varphi$ is injective on $K_{\mu} \backslash \mathcal{N}$.

Proof. $((2) \Longrightarrow(1))$. Letting $A \subset K_{\mu}$ be a Borel subset of $\mathbb{R}^{d}$, then $\chi_{A}$, the indicator function of $A$, satisfies the following condition: There exists $h \in L^{2}(\nu)$ such that $\chi_{A}(x)=$ $h(\varphi(x)) \mu$-a.e. Furthermore, we may assume that $h \geq 0 \nu$-a.e by replacing $h$ by $|h|$ if necessary. Noting that

$$
\nu(\{h \neq 0 \text { or } 1\})=\mu\left(\left\{x \in K_{\mu}, h(\varphi(x)) \neq 0 \text { or } 1\right\}\right)=\mu\left(\left\{x \in K_{\mu}, \chi_{A}(x) \neq 0 \text { or } 1\right\}\right)=0,
$$

we obtain that $h=\chi_{E}$ a.e. $(d \nu)$ where $E=\left\{y \in \mathbb{R}^{d}, h(y)=1\right\}$. Thus

$$
\chi_{A}(x)=\chi_{E}(\varphi(x))=\chi_{\varphi^{-1}(E)}(x)
$$

for $\mu$-a.e. $x$. In other words, $\chi_{A}=\chi_{\varphi^{-1}(E)}$ and (1) follows since the linear span of the characteristic functions of the Borel subsets (i.e. all simple functions) forms a dense subspace in $L^{2}(\mu)$.

$((1) \Longrightarrow(2))$. Suppose that $(1)$ holds. For a fixed vector $f \in L^{2}(\mu)$, there exists a sequence $\left\{f_{n}\right\}_{n \in \mathbb{N}}$ of the form

$$
f_{n}(x)=\sum_{j=1}^{M(n)} c_{n, j} \chi_{\varphi^{-1}\left(E_{n, j}\right)}(x)=\sum_{j=1}^{M(n)} c_{n, j} \chi_{E_{n, j}}(\varphi(x)), \quad n \geq 1,
$$

where $c_{n, j} \in \mathbb{C}$ and $E_{n, j}$ are Borel subset of $\mathbb{R}^{d}$ for $n \geq 1$ and $1 \leq j \leq M(n)$, such that $f_{n} \rightarrow f$ in $L^{2}(\mu)$. Letting

$$
h_{n}(y)=\sum_{j=1}^{M(n)} c_{n, j} \chi_{E_{n, j}}(y), \quad n \geq 1, \quad\left(y \in \mathbb{R}^{d}\right),
$$


we obtain

$\int_{\mathbb{R}^{d}}\left|h_{n}(y)-h_{m}(y)\right|^{2} d \nu(y)=\int\left|h_{n}(\varphi(x))-h_{m}(\varphi(x))\right|^{2} d \mu(x)=\int\left|f_{n}(x)-f_{m}(x)\right|^{2} d \mu(x)$.

Since $\left\{f_{n}\right\}_{n \in \mathbb{N}}$ is a Cauchy sequence in $L^{2}(\mu)$, it follows that $\left\{h_{n}\right\}_{n \in \mathbb{N}}$ is a Cauchy sequence in $L^{2}(\nu)$. Since $L^{2}(\nu)$ is complete, there exists a vector $h \in L^{2}(\nu)$ such that $h_{n} \rightarrow h$ in $L^{2}(\nu)$. Consequently,

$$
\begin{aligned}
& \int|f(x)-h(\varphi(x))|^{2} d \mu(x) \\
& \leq 2\left\{\int\left|f(x)-h_{n}(\varphi(x))\right|^{2} d \mu(x)+\int_{[0,1)^{d}}\left|h_{n}(\varphi(x))-h(\varphi(x))\right|^{2} d \mu(x)\right\} \\
& \left.\left.=\left.2\left\{\int \mid f(x)-f_{n}(x)\right)\right|^{2} d \mu(x)+\int_{\mathbb{R}^{d}} \mid h_{n}(y)\right)-\left.h(y)\right|^{2} d \nu(y)\right\}
\end{aligned}
$$

and the last quantity converges to zero as $n \rightarrow \infty$. This shows that $f(x)=h(\varphi(x))$, for $\mu$-a.e.

$((2) \Longrightarrow(3)$.) Assuming that (2) holds, for fixed $k \in\{1, \cdots, d\}$, there exist functions $h_{k}$ in $L^{2}(\nu)$ such that $x_{k}=h_{k}(\varphi(x))$, for $\mu$-a.e. $x \in \mathbb{R}^{d}$. Note that $\nu$ is a finite Borel measure and hence regular. For a regular Borel measure, we can find Borel measurable function that are equal to $h_{k} \mu$-a.e. We may therefore assume that $h_{k}$ is Borel measurable. Defining $\mathbf{h}=\left(h_{1}, \ldots, h_{d}\right)$, we obtain

$$
x=\mathbf{h}(\varphi(x))
$$

for a.e. $x \in K_{\mu}$. Consequently, letting $\mathcal{N}$ be the subset of $K_{\mu}$ for which (2.1) does not hold, it follows that $\mu\left(K_{\mu} \backslash \mathcal{N}\right)=0$. If $x, y \in K_{\mu} \backslash \mathcal{N}$ and $\varphi(x)=\varphi(y)$, applying (2.1), we obtain that $x=y$. Hence, $\varphi$ is injective on $K_{\mu} \backslash \mathcal{N}$. Finally, since $h_{k}$ and $\varphi$ are Borel measurable, so is $g=h \circ \varphi-I$, where $I$ is the identity map. $\mathcal{N}$ is therefore a Borel set since we can write

$$
K_{\mu} \backslash \mathcal{N}=\{g=0\}=\bigcap_{n=1}^{\infty}\left\{-\frac{1}{n}<g<\frac{1}{n}\right\}
$$

$\left((3) \Longrightarrow(2)\right.$.) As $\varphi$ is Borel measurable and $\left.\varphi\right|_{K_{\mu} \backslash \mathcal{N}}$ is injective, by [17, Corollary 15.2], $\varphi(B)$ is Borel measurable for all Borel sets $B \subset K_{\mu} \backslash \mathcal{N}$. Hence, we can define $g=\varphi^{-1}: \varphi\left[K_{\mu} \backslash \mathcal{N}\right] \rightarrow K_{\mu} \backslash \mathcal{N}$ such that $x=g(\varphi(x))$ and $g$ is Borel measurable. Given any $f \in L^{2}(\mu)$, since $\mu$ is a regular Borel measure, we can find a Borel measurable function $\widetilde{f}$ such that $f(x)=\widetilde{f}(x) \mu$-a.e. We also have $\widetilde{f}(x)=\widetilde{f}(g(\varphi(x)))$ on $K_{\mu} \backslash \mathcal{N}$ (thus $\mu$-a.e.). Define $h=\widetilde{f} \circ g$. Then $f(x)=\widetilde{f}(x)=h(\varphi(x)) \mu$ a.e, $h$ is Borel measurable and

$$
\int|h|^{2} d \nu=\int|\widetilde{f}(g(\varphi(x)))|^{2} d \mu(x)=\int|\widetilde{f}(x)|^{2} d \mu(x)=\int|f|^{2} d \mu .
$$

Therefore, $h \in L^{2}(\nu)$ and the proof is complete. 


\section{Main Characterization}

The aim of this section is to provide necessary and sufficient conditions for the completeness, spectrality and frame-spectrality of $E(\Lambda, \varphi)$ in terms of the essential injectivity of $\varphi$ and the corresponding property of the system $E(\Lambda)$ in the $L^{2}$-space associated with the push-forward measure.

3.1. Completeness. We recall that a collection $\left\{f_{n}\right\}$ is complete in the Hilbert space $H$ if $\left\langle f, f_{n}\right\rangle=0$ for all $n$ implies that $f=0$ on $H$. This condition is also equivalent to the fact that the closure of the linear span of $f_{n}$ is dense in $H$. We notice that an orthogonal basis must be a frame, and a frame must be complete in $H$.

Theorem 3.1. Let $\mu$ be a finite Borel measure on $\mathbb{R}^{d}$, let $\Lambda \subset \mathbb{R}^{d}$ be countable and let $\varphi: K_{\mu} \rightarrow \mathbb{R}^{d}$ be Borel measurable. Let $\nu$ be the pushforward Borel measure on $\mathbb{R}^{d}$ associated with $\varphi$. Then, the following are equivalent.

(1) The collection $E(\Lambda, \varphi)$ is complete in $L^{2}(\mu)$.

(2) $\varphi$ is $\mu$-essentially injective, and $E(\Lambda)$ is complete in $L^{2}(\nu)$.

Proof. Let us assume that (2) holds and let $g \in L^{2}(\mu)$ such that

$$
\int g(x) e^{-2 \pi i \lambda \cdot \varphi(x)} d \mu(x)=0, \quad \lambda \in \Lambda .
$$

We aim to show that $g$ must be the trivial element in $L^{2}(\mu)$. Since $\varphi$ is $\mu$-essentially injective, appealing to Lemma 2.2, there exists $h \in L^{2}(\nu)$ such that $g(x)=h(\varphi(x)) \mu$-a.e. As such,

$$
0=\int h(\varphi(x)) e^{-2 \pi i \lambda \cdot \varphi(x)} d \mu(x)=\int_{\mathbb{R}^{d}} h(y) e^{-2 \pi i \lambda \cdot y} d \nu(y), \quad \lambda \in \Lambda .
$$

By assumption, $\left\{e^{2 \pi i \lambda \cdot x}\right\}_{\lambda \in \Lambda}$ is complete in $L^{2}(\nu)$, and it follows that $h=0$. Consequently,

$$
\|g\|_{2, \mu}^{2}=\int|h(\varphi(x))|^{2} d \mu(x)=\int|h(y)|^{2} d \nu(y)=\|h\|_{2, \nu}^{2}=0
$$

and we conclude that $g=0$ as desired.

Conversely, let $\xi \in \mathbb{R}^{d}$ and fix $\epsilon>0$. Since simple functions are dense in $L^{2}(\nu)$, there exist Borel measurable sets $F_{k} \subset \mathbb{R}^{d}$ and $c_{k} \in \mathbb{C}, k=1, \ldots, m$, such that

$$
\left\|e^{2 \pi i \xi \cdot x}-\sum_{k=1}^{m} c_{k} \chi_{F_{k}}(x)\right\|_{L^{2}(\nu)}<\epsilon
$$

This implies that

$$
\int\left|e^{2 \pi i \xi \cdot \varphi(x)}-\sum_{k=1}^{m} c_{k} \chi_{\varphi^{-1}\left(F_{k}\right)}(x)\right|^{2} d \mu(x)=\int\left|e^{2 \pi i \xi \cdot \varphi(x)}-\sum_{k=1}^{m} c_{k} \chi_{F_{k}}(\varphi(x))\right|^{2} d \mu(x)<\epsilon^{2} .
$$

Since we assume that the span of the collection $E(\Lambda, \varphi)=\left\{e^{2 \pi i \lambda \cdot \varphi(x)}\right\}_{\lambda \in \Lambda}$ is dense in $L^{2}(\mu)$, the above shows that the span of the collection $\chi_{\varphi^{-1}(F)}$, where $F \subset \mathbb{R}^{d}$ is Borel, is also dense and therefore $\varphi$ is $\mu$-essentially injective by Lemma 2.2. We finally show that $E(\Lambda)$ is complete in $L^{2}(\nu)$. To see this, let $f \in L^{2}(\mu)$ and suppose that $\int f(x) e^{-2 \pi i \lambda \cdot x} d \nu(x)=0$ for all $\lambda \in \Lambda$. Then

$$
\int f(\varphi(x)) e^{-2 \pi i \lambda \cdot \varphi(x)} d \mu(x)=0, \forall \lambda \in \Lambda \text {. }
$$


By (1), $f(\varphi(x))=0 \mu$-a.e. As a set, we note that $\varphi^{-1}\{y: f(y) \neq 0\}=\{x: f(\varphi(x)) \neq 0\}$.

$$
\nu(\{f \neq 0\})=\mu\left(\varphi^{-1}\{f \neq 0\}\right)=\mu(\{x: f(\varphi(x)) \neq 0\})=0 .
$$

This shows $f=0, \nu$-a.e, which means that $E(\Lambda)$ is complete in $L^{2}(\nu)$.

3.2. Orthogonal basis and frames. We shall now give a complete characterization of functions $\varphi: K_{\mu} \rightarrow \mathbb{R}^{d}$ for which $E(\Lambda, \varphi)$ forms an orthogonal basis or a frame for $L^{2}(\mu)$.

Theorem 3.2. Let $\mu$ be a finite Borel measure, $\Lambda \subset \mathbb{R}^{d}$ be countable and let $\varphi: K_{\mu} \rightarrow \mathbb{R}^{d}$ be Borel measurable. Let $\nu$ be the pushforward Borel measure on $\mathbb{R}^{d}$ associated with $\varphi$ and $\mu$ Then

(1) The collection $E(\Lambda, \varphi)$ is an orthogonal basis for $L^{2}(\mu)$ if and only if $\varphi$ is $\mu$ essentially injective and $\nu$ is a spectral measure with a spectrum $\Lambda$.

(2) The collection $E(\Lambda, \varphi)$ forms a frame for $L^{2}(\mu)$ if and only if $\varphi$ is $\mu$-essentially injective and $\nu$ is a frame-spectral measure with a frame spectrum $\Lambda$.

Proof. For the first part, we note that the pushforward Borel measure $\nu$ is bounded and

$$
\int_{\mathbb{R}^{d}} f(y) d \nu(y)=\int f(\varphi(x)) d \mu(x)
$$

for any bounded, continuous function $f: \mathbb{R}^{d} \rightarrow \mathbb{C}$. The orthogonality in $L^{2}(\nu)$ of the collection of exponentials with spectrum in $\Lambda$ gives

$$
\int e^{2 \pi i\left(\lambda-\lambda^{\prime} \cdot \varphi(x)\right)} d \mu(x)=\int_{\mathbb{R}^{d}} e^{2 \pi i\left(\lambda-\lambda^{\prime}\right) \cdot y} d \nu(y)=\delta_{\lambda, \lambda^{\prime}}, \quad \lambda, \lambda^{\prime} \in \Lambda .
$$

This shows that the collection $\left\{e^{2 \pi i \lambda \cdot \varphi(x)}\right\}_{\lambda \in \Lambda}$ is mutually orthogonal for $L^{2}(\mu)$ if and only if $\left\{e^{2 \pi i \lambda \cdot x}\right\}_{\lambda \in \Lambda}$ is mutually orthogonal for $L^{2}(\nu)$. Next, appealing to Theorem 3.1. we obtain that $E(\Lambda, \varphi)$ is complete if and only if $\varphi$ is $\mu$-essentially injective and $E(\Lambda)$ is complete in $L^{2}(\nu)$. This takes care of the first part of the result.

For the second part, let us suppose that $E(\Lambda, \varphi)$ forms a frame for $L^{2}(\mu)$. As such, the collection $E(\Lambda, \varphi)$ is complete in $L^{2}(\mu)$. By Theorem 3.1, $\varphi$ is $\mu$-essentially injective. Next, given $h \in L^{2}(\nu)$,

$$
\sum_{\lambda \in \Lambda}\left|\int h(y) e^{-2 \pi i \lambda \cdot y} d \nu(y)\right|^{2}=\sum_{\lambda \in \Lambda}\left|\int h(\varphi(x)) e^{-2 \pi i \lambda \cdot \varphi(x)} d \mu(y)\right|^{2} .
$$

However,

$$
\int|h(y)|^{2} d \nu(y)=\int|h(\varphi(x))|^{2} d \mu(x),
$$

and the frame inequalities for $E(\Lambda)$ in $L^{2}(\nu)$ follow from those of $E(\Lambda, \varphi)$ in $L^{2}(\mu)$. Conversely, since $\varphi$ is $\mu$-essentially injective, any function $f \in L^{2}(\mu)$ can be written as $f(x)=h(\varphi(x)) \mu$-a.e. with $h \in L^{2}(\nu)$. Therefore, the frame inequalities for $E(\Lambda, \varphi)$ for $L^{2}(\mu)$ also follow from those of $E(\Lambda)$ in $L^{2}(\nu)$. 
3.3. Lebesgue measures on general sets. We now turn to study the special case where $\mu$ is the Lebesgue measure restricted on a set. Given a Borel set $\Omega \subseteq \mathbb{R}^{d}$ of positive finite Lebesgue measure, we define the measure $m_{\Omega}$ as follows:

$$
m_{\Omega}=\chi_{\Omega} d x
$$

Letting $\Lambda$ be a countable discrete set, we define the upper and lower Beurling density of $\Lambda$, denoted by $D^{+}(\Lambda)$ and $D^{-}(\Lambda)$ respectively, as follows:

$$
D^{+}(\Lambda)=\limsup _{R \rightarrow \infty} \sup _{x \in \mathbb{R}^{d}} \frac{\#\left(\Lambda \cap Q_{R}(x)\right)}{R^{d}} \text {, and } D^{-}(\Lambda)=\liminf _{R \rightarrow \infty} \inf _{x \in \mathbb{R}^{d}} \frac{\#\left(\Lambda \cap Q_{R}(x)\right)}{R^{d}},
$$

where $Q_{R}(x)=x+\left[-\frac{R}{2}, \frac{R}{2}\right]^{d}$ denotes the hypercube of side length $R$ centered at $x$. In [13, Proposition 2.1], He, Lai and Lau proved

Proposition 3.3. If $D^{-}(\Lambda)>0$ and $\Lambda$ is a frame spectrum for $L^{2}(\mu)$, then $\mu$ must be absolutely continuous with respect to the Lebesgue measure.

Moreover, in [5, Theorem 1.3 and Corollary 1.4], the following was proved.

Theorem 3.4. Let $\Lambda$ be a countable discrete set with $D^{-}(\Lambda)>0$ and let $\mu$ be a finite Borel measure. The following holds true

(1) If $\left\{e^{2 \pi i \lambda \cdot x}: \lambda \in \Lambda\right\}$ forms a frame for $L^{2}(\mu)$, then $\mu$ is absolutely continuous with respect to the Lebesgue measure with density $g$ satisfying $0<m \leq g \leq M<\infty$ almost everywhere on the set $\{g \neq 0\}$. (for some positive real numbers $m$ and $M$.)

(2) If $\left\{e^{2 \pi i \lambda \cdot x}: \lambda \in \Lambda\right\}$ forms an orthogonal basis for $L^{2}(\mu)$, then $\mu$ is absolutely continuous with respect to the Lebesgue measure with a constant density.

We now have the following characterization.

Theorem 3.5. Let $\mu=m_{\Omega}$ where $\Omega$ is a measurable set of finite positive Lebesgue measure and let $\Lambda \subset \mathbb{R}^{d}$ be countable with $D^{-}(\Lambda)>0$. Given a Borel measurable function $\varphi: K_{\mu} \rightarrow$ $\mathbb{R}^{d}$, define $\nu=\varphi_{*} \mu$ to be the pushforward of the measure $\mu$ via $\varphi$. Then, the following holds.

(1) The collection $\left\{e^{2 \pi i \lambda \cdot \varphi(x)}\right\}_{\lambda \in \Lambda}$ is an orthogonal basis for $L^{2}(\mu)$ if and only if there exists a set $\Omega_{0}$ such that $\varphi$ is injective on $\Omega_{0}$ and $m\left(\Omega \backslash \Omega_{0}\right)=0$ with

(a) $\varphi$ is injective on $\Omega_{0}$,

(b) $\varphi_{*} m_{\Omega_{0}}=c \cdot m_{\varphi\left(\Omega_{0}\right)}$ for some $c>0$.

(c) $\varphi\left(\Omega_{0}\right)$ is a spectral set with a spectrum $\Lambda$.

(2) The collection $\left\{e^{2 \pi i \lambda \cdot \varphi(x)}\right\}_{\lambda \in \Lambda}$ forms a frame for $L^{2}(\mu)$ if and only if there exists a set $\Omega_{0}$ such that $\varphi$ is injective on $\Omega_{0}$ and $m\left(\Omega \backslash \Omega_{0}\right)=0$ with

(a) $\varphi$ is injective on $\Omega_{0}$,

(b) There exists $0<m \leq M<\infty$ such that $\varphi_{*} \mu=g(x) d x$ with $m \leq g \leq M$ a.e. on $\varphi\left(\Omega_{0}\right)$ and

(c) $\left\{e^{2 \pi i \lambda \cdot x}\right\}_{\lambda \in \Lambda}$ forms a Fourier frame for $L^{2}\left(\varphi\left(\Omega_{0}\right)\right)$.

Proof. In order to prove the first statement, note that, by Theorem 3.2, the fact that the collection $\left\{e^{2 \pi i \lambda \cdot \varphi(x)}\right\}_{\lambda \in \Lambda}$ forms an orthogonal basis for $L^{2}(\mu)$ is equivalent to the essential injectivity of $\varphi$ and the fact that $\varphi_{*} m_{\Omega}$ is a spectral measure with spectrum $\Lambda$. Thus the conditions in 1(a), 1(b) and 1(c) clearly imply that $\left\{e^{2 \pi i \lambda \cdot \varphi(x)}\right\}_{\lambda \in \Lambda}$ is an orthogonal basis for $L^{2}(\mu)$.

Conversely, according to Theorem 3.2 again, if that same collection is an orthogonal basis for $L^{2}(\mu), \varphi$ must be $\mu$-essentially injective, i.e. 1(a) holds and denote by $\Omega_{0}$ to 
be the set of full measure so that $\varphi$ is injective on. Note that the second condition in Theorem 3.2 implies that $\varphi_{*} m_{\Omega_{0}}$ must be a spectral measure with spectrum $\Lambda$. Since we are also assuming that $D^{-}(\Lambda)>0$, part (2) of Theorem 3.4 then shows that, for some $c>0, \varphi_{*} m_{\Omega_{0}}=c \cdot m_{D}$, where $D$ is an essential support of $\varphi_{*} m_{\Omega_{0}}$. But it is direct to see that $\varphi\left(\Omega_{0}\right)$ is a support of $\varphi_{*} m_{\Omega_{0}}$ since

$$
\varphi_{*} m_{\Omega_{0}}\left(\varphi\left(\Omega_{0}\right)\right)=m_{\Omega_{0}}\left(\varphi^{-1} \varphi\left(\Omega_{0}\right)\right)=m\left(\Omega_{0}\right)=\varphi_{*} m_{\Omega_{0}}\left(\mathbb{R}^{d}\right) .
$$

The proof of (2) is similar to (1) by invoking Theorem 3.4 (2). We will omit the details.

It turns out that the condition on the Beurling density cannot be removed. If we do not restrict the density of $\Lambda$, the measure $\varphi_{*} m_{\Omega}$ may be singular with respect to the Lebesgue measure and the space $L^{2}(\Omega)$ could actually admit an orthonormal basis of exponentials with non-linear phases associated with a spectrum $\Lambda$ possessing a zero Beurling density (See Example 4.1).

\section{ExAmples And Illustration}

In this section, we present some examples of exponentials with non-linear phases that can form either a basis or a a frame for the Hilbert space $L^{2}(\mu)$.

4.1. Fractal examples. Recall that the standard middle-fourth Cantor measure $\nu_{4}$ is the unique measure supported on the middle-fourth Cantor set $K_{4}$ satisfying the self-similar identity.

$$
\nu_{4}(E)=\frac{1}{2} \nu_{4}(4 E)+\frac{1}{2} \nu_{4}(4 E-2), \quad E \subset \mathbb{R}, \quad E \text { Borel. }
$$

For this self-similar measure, Jorgensen and Pedersen [16] found that $L^{2}\left(\nu_{4}\right)$ admits an orthonormal basis basis of exponentials $\left\{e^{2 \pi i \lambda x}: \lambda \in \Lambda\right\}$, where

$$
\Lambda_{4}=\left\{\sum_{i=0}^{n-1} 4^{i} a_{i}: a_{i} \in\{0,1\}, n=1,2,3 \ldots\right\},
$$

showing thus that $\nu_{4}$ is a spectral measure. The support of $\nu_{4}, K_{4}$, can be expressed as the set

$$
K_{4}=\bigcap_{n=1}^{\infty} \bigcup_{j=1}^{2^{n}} I_{j, n}
$$

where $I_{j, n}$ are the basic intervals of the Cantor set.

Example 4.1. Let $\mu$ be the Lebesgue measure on $[0,1]$ and let $\varphi:[0,1] \rightarrow K_{4}$ be the map

$$
\varphi\left(\sum_{i=1}^{\infty} \frac{\epsilon_{i}}{2^{i}}\right)=\sum_{i=1}^{\infty} \frac{2 \epsilon_{i}}{4^{i}},
$$

where $\epsilon_{i} \in\{0,1\}$. This map is well-defined except on the set of dyadic rational numbers, which has measure zero. We note that for each $n^{\text {th }}$ basic interval $I_{j, n}, \varphi^{-1}\left(I_{j, n}\right)$ is a dyadic interval. The collection of all the preimages of the $n^{\text {th }}$ basic intervals, $\varphi^{-1}\left(I_{j, n}\right)$, $j=1, \ldots, 2^{n}$ are exactly all the dyadic intervals at the $n^{\text {th }}$ stage. Therefore, Lemma 2.2(1) is satisfied, and thus, $\varphi$ is $\mu$-essentially injective. Moreover, as the measure $\nu_{4}$ and $\mu$ are completely determined by their values on the basic intervals, we must have

$$
\varphi_{*} \mu=\nu_{4}
$$


Hence, by Theorem 3.2 , we have that

$$
\left\{e^{2 \pi i \lambda \varphi(x)}: \lambda \in \Lambda_{4}\right\}
$$

is an orthonormal basis for $L^{2}[0,1]$.

Example 4.2. (Non-linear phased exponential bases for middle-third Cantor measures) Let $K_{3}$ be the middle-third Cantor set and $\nu_{3}$ be the middle-third Cantor measure, which can be defined analogously by replacing 4 with 3 in the middle-fourth Cantor set definition. Jorgenesen and Pedersen [16] proved that there is no exponential orthonormal basis for $L^{2}\left(\nu_{3}\right)$. Nonetheless, we can define

$$
\varphi\left(\sum_{i=1}^{\infty} \frac{\epsilon_{i}}{3^{i}}\right)=\sum_{i=1}^{\infty} \frac{\epsilon_{i}}{4^{i}},
$$

Using a similar proof in Example 4.1, we obtain that we have that

$$
\left\{e^{2 \pi i \lambda \varphi(x)}: \lambda \in \Lambda_{4}\right\}
$$

is an orthonormal basis for $L^{2}\left(\nu_{3}\right)$. This example was also observed earlier in [?].

4.2. Unit balls. Constructing an exponential Riesz basis for the open unit disc $\mathbb{D}$ has been a challenging problem in basis and frame theory. We are however, able to offer an explicit non-linear phased exponential family forming an orthonormal basis for $L^{2}(\mathbb{D})$. Let $A, B$ be open sets on $\mathbb{R}^{d}$. We say that $\varphi: A \rightarrow B$ is a $C^{1}$-diffeomorphism if $\varphi$ is a $C^{1}$ bijective map from $A$ to $B . \varphi$ is called measure-preserving if $\varphi_{*} m_{A}=m_{B}$. We need the following simple proposition.

Proposition 4.3. Let $A, B$ be open sets on $\mathbb{R}^{d}$ and let $\varphi: A \rightarrow B$ be a $C^{1}$ diffeomorphism. Let $J(\varphi)$ be the Jacobian matrix of $\varphi$. Then $\varphi_{*} m_{A}=m_{B}$ if and only if $|\operatorname{det} J(\varphi)|=1$.

Proof. We note that the change of variable formula is now valid. Therefore, we have

$$
\varphi_{*} m_{A}(E)=\int_{A} \chi_{E}(\varphi(x)) d x=\int \chi_{E}(\varphi(x)) \chi_{B}(\varphi(x)) d x=\int \chi_{E \cap B}(x)|\operatorname{det} J(\varphi(x))|^{-1} d x .
$$

Hence, if $|\operatorname{det} J(\varphi)|=1$, then $\varphi_{*} m_{A}=m_{B}$ holds. Conversely, if $\varphi_{*} m_{A}=m_{B}$ holds, then

$$
\int \chi_{E \cap B}(x)\left(|\operatorname{det} J(\varphi(x))|^{-1}-1\right)=0
$$

for all Borel sets $E$. Hence, $|\operatorname{det} J(\varphi(x))|^{-1}=1$ holds a.e. on $B$.

It is possible to have a measure preserving $C^{1}$ diffeomorphism between $\mathbb{D}$ and the $\ell^{1}$ ball (which is a square) with the same area as $\mathbb{D}$. The map was given by [15, p.160] in which $\varphi(x, y)=(X, Y)$ where

$$
\begin{aligned}
& X=\operatorname{sgn}(x) \frac{\sqrt{x^{2}+y^{2}}}{\sqrt{2 \pi}}\left(\frac{\pi}{2}+\sin ^{-1}\left(\frac{x^{2}-y^{2}}{x^{2}+y^{2}}\right)\right), \\
& Y=\operatorname{sgn}(y) \frac{\sqrt{x^{2}+y^{2}}}{\sqrt{2 \pi}}\left(\frac{\pi}{2}-\sin ^{-1}\left(\frac{x^{2}-y^{2}}{x^{2}+y^{2}}\right)\right) .
\end{aligned}
$$

Note that if $x^{2}+y^{2}=1$, then $|X|+|Y|=\sqrt{\pi / 2}$. Hence, the $\ell^{1}$-ball formed has a measure of $\pi$. Using this map, Theorem 3.2 and the fact that square admits an exponential orthonormal basis $E(\Lambda)$. We have thus proved that 
Proposition 4.4. There exists a map $\varphi$ and a set $\Lambda$ such that $E(\Lambda, \varphi)$ form an orthonormal basis for $L^{2}(\mathbb{D})$.

\section{Classification of Continuous phase functions where $d=1$}

In previous sections, we characterized Borel measurable function $\varphi$ so that $E(\Lambda, \varphi)$ forms a basis or frame. From this section on, we will focus our attention on $\varphi$ being continuous functions or differentiable functions. In particular, we are interested in the case where $\Lambda=\mathbb{Z}^{d}$ and $E(\Lambda, \varphi)$ forms an orthogonal basis for $[0,1]^{d}$.

We will first study $d=1$ in this section. Here, we can work on $\varphi$ being continuous with a mild assumption that $\varphi$ preserves measure zero sets. We may also assume that $\varphi(0)=0$ since otherwise, the exponentials will just be differing by a phase factor $e^{2 \pi i \lambda \cdot \varphi(0)}$, which will not affect the basis property. Our main result is as follows:

Theorem 5.1. Let $\varphi:[0,1] \rightarrow \mathbb{R}$ be a continuous function that maps Lebesgue measure zero sets to measure zero sets. Suppose also that $\varphi(0)=0$. Then $E(\mathbb{Z}, \varphi)$ is an orthonormal basis for $L^{2}[0,1]$ if and only if $\varphi(x)= \pm x$.

We begin our proof with a general lemma.

Lemma 5.2. Let $K$ be a bounded set of positive Lebesgue measure and let $\varphi: K \rightarrow \mathbb{R}^{d}$ be a continuous function taking measure zero sets to measure zero sets. Suppose there exists a set $E \subset \mathbb{R}^{d}$, of positive measure satisfying $E=\varphi\left(U_{1}\right)=\varphi\left(U_{2}\right)$ with $U_{1} \cap U_{2}=\emptyset$ and $U_{1}, U_{2}$ are of positive Lebesgue measure in $K$. Then $\varphi$ cannot be $\mu$-essentially injective.

Proof. We argue by contradiction. Suppose that $\varphi$ is $\mu$-essentially injective. Letting $f(x)=x$, we have then $f \in L^{2}(K)$. Since $\varphi$ is $\mu$-essentially injective, one can find $h \in L^{2}(\nu)$, where $\nu=\varphi_{*} \mu$, such that $x=h(\varphi(x))$ for a.e. $x \in K$. Let $E, U_{1}, U_{2}$ be sets satisfying the conditions in the statement of the lemma. Then

$$
\mathcal{F}_{i}=\left\{x \in U_{i}: h(\varphi(x))=x\right\}
$$

has full Lebesgue measure in $U_{i}$ for $i=1,2$. Consider the following sets:

$$
\mathcal{K}_{1}=\varphi\left(\mathcal{F}_{1}\right), \mathcal{K}_{2}=\varphi\left(\mathcal{F}_{2}\right) .
$$

Since $\varphi$ maps measure zero sets to measure zero sets, $\mathcal{K}_{1}$ and $\mathcal{K}_{2}$ have full Lebesgue measure in $E$. Moreover, $\varphi$ is also continuous, so $\mathcal{K}_{i}$ are measurable ( since we can decompose $\mathcal{F}_{i}$ into $F_{\sigma}$-sets and measure zero sets). Thus, $m\left(\mathcal{K}_{1} \cap \mathcal{K}_{2}\right)=m(E)$. Next, given $a \in \mathcal{K}_{1} \cap \mathcal{K}_{2}$, it is clear that $a=\varphi(x)=\varphi(y)$ for some $x \in \mathcal{F}_{1} \subset U_{1}$ and $y \in \mathcal{F}_{2} \subset U_{2}$. Hence,

$$
x=h(\varphi(x))=h(a)=h(\varphi(y))=y,
$$

and this contradicts the fact that $U_{1}$ and $U_{2}$ are disjoint sets. This completes the proof of the lemma.

Proposition 5.3. Let $\varphi:[0,1] \rightarrow \mathbb{R}$ be a continuous function mapping measure zero sets to measure zero sets. If $\varphi$ is $\mu$-essentially injective, then $\varphi$ is injective.

Proof. We first observe that $\varphi$ cannot be a constant function on any non-degenerate subinterval $I \subset[0,1]$. Otherwise, let $h \in L^{2}(\nu)$, where $\nu=\varphi_{*} \mu$, such that $x=h(\varphi(x))$ a.e. on $[0,1]$. If $\varphi(x)=c$ for $x \in I$, we would obtain that $h(c)=x$ for almost every $x \in I \subset[0,1]$, clearly a contradiction. 
Suppose that $\varphi$ is not injective. There exist $x_{0}, y_{0}$ such that $x_{0} \neq y_{0}$ and $\varphi\left(x_{0}\right)=$ $\varphi\left(y_{0}\right)=c$. As $\varphi$ is continuous, given any $\epsilon>0$, we can find $\delta>0$ such that $\varphi(x) \epsilon$ $(c-\epsilon, c+\epsilon)$ and $\varphi(y) \in(c-\epsilon, c+\epsilon)$ whenever $\left|x-x_{0}\right|<\delta$ and $\left|y-y_{0}\right|<\delta$. Let $x_{1} \in\left(x_{0}-\delta, x_{0}+\delta\right)$ and $y_{1} \in\left(y_{0}-\delta, y_{0}+\delta\right)$. For sufficiently small $\delta$, we may assume that $\left(x_{0}-\delta, x_{0}+\delta\right)$ and $\left(y_{0}-\delta, y_{0}+\delta\right)$ are disjoint. Since $\varphi$ cannot be constant on any intervals, we may assume that $\varphi\left(x_{1}\right), \varphi\left(y_{1}\right)$ are not equal to $c$ and $\varphi\left(x_{1}\right)<\varphi\left(y_{1}\right)$. For the subsequent analysis, we define $I_{x, y}$ and $I_{x, y}^{\circ}$ respectively as the closed and open interval with endpoints $x, y$.

Case (1): $\varphi\left(x_{1}\right)<\varphi\left(y_{1}\right)<c$ or $c<\varphi\left(x_{1}\right)<\varphi\left(y_{1}\right)$. Since these two cases are symmetric, there is no loss of generality in only addressing one of them. By the intermediate value theorem applied to $\varphi$ defined on $I_{x_{1}, x_{0}}, \varphi$ assumes all values in the interval $\left[\varphi\left(x_{1}\right), c\right]$ from $I_{x_{1}, x_{0}}$. Hence, $\varphi\left(I_{x_{1}, x_{0}}\right) \supset\left[\varphi\left(x_{1}\right), c\right] \supset\left[\varphi\left(y_{1}\right), c\right]$. Similarly, we also have $\varphi\left(I_{y_{1}, y_{0}}\right) \supset$ $\left[\varphi\left(y_{1}\right), c\right]$. Note that $\left[\varphi\left(y_{1}\right), c\right]$ is now a non-degenerate subinterval of $\left[\varphi\left(x_{1}\right), c\right]$. Let $E=$ $\left(\varphi\left(y_{1}\right), c\right)$ and let $U_{1}=I_{x_{1}, x_{0}}^{\circ} \cap \varphi^{-1}(E), U_{2}=I_{y_{1}, y_{0}}^{\circ} \cap \varphi^{-1}(E)$. Then $U_{1}, U_{2}$ has positive Lebesgue measure (since they are open) and disjoint. Moreover, $\varphi\left(U_{1}\right)=\varphi\left(U_{2}\right)=E$. All assumptions in Lemma 5.2 are satisfied. Hence, $\varphi$ cannot be $\mu$-essentially injective. This completes the proof of Case (1).

Case (2): $\varphi\left(x_{1}\right)<c<\varphi\left(y_{1}\right)$. We may assume that $\varphi\left[x_{0}-\delta, x_{0}+\delta\right] \subset[c-\epsilon, c]$ and $\varphi\left[y_{0}-\delta, y_{0}+\delta\right] \subset[c, c+\epsilon]$. Otherwise, we can select $x_{1}, y_{1}$ to satisfy the assumptions in Case (1). We can also assume that the endpoints do not take the value $c$, otherwise, we choose a smaller $\delta$. By the intermediate value theorem, $\varphi\left(I_{x_{1}, x_{0}}\right) \supset\left[\varphi\left(x_{1}\right), c\right]$. On the other hand, consider the interval $\left(x_{0}+\delta, y_{0}-\delta\right)$ if $x_{0}<y_{0}$ and $\left(y_{0}+\delta, x_{0}-\delta\right)$ if $y_{0}<x_{0}$. We only consider the first case since the case $y_{0}<x_{0}$ is similar. Intermediate Value Theorem tells us that all values in the interval $\varphi\left[x_{0}+\delta, y_{0}-\delta\right] \supset\left[\varphi\left(x_{0}+\delta\right), \varphi\left(y_{0}-\delta\right)\right]$. Note that the interval $E=\left(\max \left\{\varphi\left(x_{1}\right), \varphi\left(x_{0}+\delta\right)\right\}, c\right)$ is non-degenerate (since endpoints do not take the value $c)$. And the set $U_{1}=I_{x_{1}, x_{0}}^{\circ} \cap \varphi^{-1}(E)$ and $U_{2}=\left(x_{0}+\delta, y_{0}-\delta\right) \cap \varphi^{-1}(E)$ satisfies the assumption that $\varphi\left(U_{1}\right)=\varphi\left(U_{2}\right)=E$ with positive Lebesgue measure. The assumption in Lemma 5.2 are all satisfied, so $\varphi$ cannot be $\mu$-essentially injective. This completes the proof.

Proof of Theorem 5.1. The statement that $\varphi(x)= \pm x$ implies that $\left\{e^{2 \pi i k \cdot \varphi(x)}: k \in \mathbb{Z}\right\}$ is an orthonormal basis for $L^{2}[0,1]$ is evident and we shall focus on its converse. So, let us assume that $E\left(\mathbb{Z}^{d}, \varphi\right)$ is an orthonormal basis for $L^{2}[0,1]$. Then $\varphi$ must be $\mu$-essentially injective by Theorem 3.2. Proposition 5.3 implies that $\varphi$ must be injective. As $\varphi$ is continuous, $\varphi$ is monotone.

Without loss of generality, we can assume $\varphi$ is increasing. Then $\varphi[0,1]=[0, \varphi(1)]$. Hence, $\varphi_{*} m_{[0,1]}=c \cdot m_{[0, \varphi(1)]}$ by Theorem $3.21(\mathrm{~b})$. As $E(\mathbb{Z})$ is an exponential orthonormal basis for $m_{[0, \varphi(1)]}$, we must have the $\varphi(1)=m([0, \varphi(1)])=1$ and $c=1$. Thus, $t=$ $m_{[0,1]}(0, t)=\varphi_{*} m_{[0,1]}((0, t))=\varphi^{-1}(t)$ and it follows that $\varphi(x)=x$. The case that $\varphi$ is decreasing is similar and $\varphi(x)=-x$.

Theorem 5.1 guarantees that under a weak condition imposed on $\varphi$, linear functions are the only maps $\varphi$ for which the system $E(\Lambda, \varphi)$ is an orthonormal basis for $L^{2}[0,1]$. As it is well-known that all Lipschitz functions map measure zero sets to measure zero sets, so Theorem 5.1 applies.

We finally remark that $\mu$-essentially injectivity and injectivity are not the same concept even if $\varphi$ is a continuous function. In search of the literature, we found that Foschini [8] constructed a continuous $\mu$-essentially injective function that is not monotone in any 
intervals via Wiener process. We do not know if this function can produce an exponential orthonormal basis with a non-linear phase. However, it shows that the assumption about preservation of measure zero sets in Proposition 5.3 cannot be removed.

\section{NON-LINEAR PHASE IN HIGHER DIMENSIONS}

6.1. A sufficient condition. In this section, we will investigate the construction of exponential orthogonal bases with non-linear phases in higher dimensions. Our investigation reveals that in the multidimensional settings, we cannot expect a result that is analogous to Theorem 5.1 (see Theorem 6.1).

Our first result in this section provides a large class of functions $\varphi$ such that $E\left(\mathbb{Z}^{d}, \varphi\right)$ is an orthonormal basis for $L^{2}\left([0,1]^{d}\right)$ for arbitrary $d$.

Theorem 6.1. Let $\varphi:[0,1]^{d} \rightarrow \mathbb{R}^{d}$ and let $x=\left(x_{1}, \ldots, x_{d}\right)$ be such that

$$
\varphi(x)=\left(x_{1}+l_{1}\left(x_{2}, \cdots x_{d}\right), x_{2}+l_{2}\left(x_{3}, \cdots, x_{d}\right), \cdots, x_{d-1}+l_{d-1}\left(x_{d}\right), x_{d}\right)
$$

for some $C^{1}$-functions $l_{1}, l_{2}, \cdots, l_{d-1}$. Then $E\left(\mathbb{Z}^{d}, \varphi\right)$ is an orthonormal basis for $L^{2}[0,1]^{d}$.

Remark 6.2. Let $M$ be a square matrix of order $d$ with integer entries satisfying $|\operatorname{det} M|=$ 1. Since $\mathbb{Z}^{d}$ is invariant under the action of the transpose of $M$, it is easy to verify that $E\left(\mathbb{Z}^{d}, M \varphi\right)$ is an orthonormal basis for $L^{2}[0,1]^{d}$ if and only if $E\left(\mathbb{Z}^{d}, \varphi\right)$ is an orthonormal basis for $L^{2}[0,1]^{d}$. More generally for arbitrary $A \in G L(d, \mathbb{R})$, the system $E\left(\mathbb{Z}^{d}, A \varphi\right)$ is an orthonormal basis for $L^{2}[0,1]^{d}$ if and only if $E\left(A^{T} \mathbb{Z}^{d}, \varphi\right)$ is an orthonormal basis for $L^{2}[0,1]^{d}$.

In setting the stage for the proof of Theorem 6.1, recall the following. Let $\Omega$ be a subset of $\mathbb{R}^{d}$. We say that $\Omega$ is a translational tile by a set $\mathcal{J}$ if

$$
m\left((\Omega+t) \cap\left(\Omega+t^{\prime}\right)\right)=0 \forall t \neq t^{\prime} \in \mathcal{J} \text { and } \bigcup_{t \in \mathcal{J}}(\Omega+t)=\mathbb{R}^{d} .
$$

When the first condition described above holds, we say that $\Omega$ is a packing set. Given a lattice $\Gamma=A\left(\mathbb{Z}^{d}\right)$ where $A$ is an invertible matrix of order $d$, the quantity $|\operatorname{det}(A)|$ is called the volume of $\Gamma$, and it is not difficult to verify that if $\Omega$ is a packing set by a lattice $\Gamma$ and $m(\Omega)=|\operatorname{det}(A)|$, then $\Omega$ tiles $\mathbb{R}^{d}$ translationally with $\Gamma$ (see e.g. [10, Theorem 2.1]). Furthermore, the following is a well-known result due to Fuglede [9]

Theorem 6.3. Let $\Gamma$ be a full-rank lattice of $\mathbb{R}^{d}$. Then $L^{2}(\Omega)$ admits an exponential orthogonal basis $E(\Gamma)$ if and only if $\Omega$ is a translational tile by the dual lattice of $\Gamma$ (i.e. $\left.\Gamma^{\perp}:=\left\{x \in \mathbb{R}^{d}: x \cdot \gamma \in \mathbb{Z} \forall \gamma \in \Gamma\right\}\right)$.

Proof of Theorem 6.1. In light of Theorem 3.2, to prove Theorem6.1, it suffices to establish the following: (i) $\varphi$ is $\mu$-essentially injective, (ii) $\varphi_{*} m_{[0,1]^{d}}=m_{\varphi\left([0,1]^{d}\right)}$ and (iii) $E\left(\mathbb{Z}^{2}\right)$ is an orthonormal basis for $L^{2}\left(\varphi\left([0,1]^{d}\right)\right.$.

For the first part, we only need to show that $\varphi$ is an injective map. Indeed, assuming that $\varphi(x)=\varphi(y)$, the last coordinates of $x$ and $y$ are equal to each other. Next, by assumption, $x_{d-1}+l_{d-1}\left(x_{d}\right)=y_{d-1}+l_{d-1}\left(y_{d}\right)$ and straightforward calculations show that $x_{d-1}=y_{d-1}$. Proceeding in this fashion, we establish $x=y$, showing that $\varphi$ is injective.

To show (ii), we first verify that the Jacobian of $\varphi$ is equal to one. Indeed, since

$$
\varphi(x)=x+\left(l_{1}\left(x_{2}, \cdots x_{d}\right), l_{2}\left(x_{3}, \cdots, x_{d}\right), \cdots, l_{d-1}\left(x_{d}\right), 0\right)
$$


and the Jacobian of $\varphi$ is a unipotent matrix. i.e. $J_{\varphi}(x)=I+N(x)$ for some matrix-valued function $N(x)$ such that it is strictly upper triangular. Hence, $J_{\varphi}$ is upper-triangular with all ones on its diagonal and $\operatorname{det} J_{\varphi}=1$. Thus, $\varphi$ defines a local diffeomorphism and is also injective, and as such $\varphi$ induces a $C^{1}$-diffeomorphism between its domain and its range. Consequently, $\varphi_{*} m_{[0,1]^{d}}=m_{\varphi\left([0,1]^{d}\right)}$.

Finally, to prove (iii), it suffices to show that (see to Theorem [6.3) $\Omega:=\varphi\left([0,1]^{d}\right)$ is a translational tile for $\mathbb{R}^{d}$. To this end, we first claim that $\left(\varphi\left([0,1)^{d}\right)+k\right) \cap \varphi\left([0,1)^{d}\right)$ is empty whenever $k$ is a nonzero element of $\mathbb{Z}^{d}$. Indeed, suppose that $x, y \in[0,1)^{d}$ such that $\varphi(x)-\varphi(y)=k \in \mathbb{Z}^{d}$. This gives the following system of equations

$$
\left\{\begin{array}{c}
k_{1}=x_{1}-y_{1}+l_{1}\left(x_{2}, \cdots x_{d}\right)-l_{1}\left(y_{2}, \cdots, y_{d}\right) \\
k_{2}=x_{2}+l_{2}\left(x_{3}, \cdots x_{d}\right)-y_{2}-l_{2}\left(y_{3}, \cdots y_{d}\right) \\
\vdots \\
k_{d-1}=x_{d-1}+l_{d-1}\left(x_{d}\right)-y_{d-1}-l_{d-1}\left(y_{d}\right) \\
k_{d}=x_{d}-y_{d}
\end{array} .\right.
$$

Since $x_{d}-y_{d} \in(-1,1) \cap \mathbb{Z}$, it must be the case that $k_{d}=0$. This, however, implies that $x_{d}=y_{d}$. Therefore,

$$
k_{d-1}=x_{d-1}+l_{d-1}\left(x_{d}\right)-y_{d-1}-l_{d-1}\left(x_{d}\right)=x_{d-1}-y_{d-1} \in(-1,1) \cap \mathbb{Z}
$$

and $x_{d-1}=y_{d-1}$. Proceeding inductively, we obtain $x=y$. This implies that $\varphi\left([0,1)^{d}\right)$ is a packing set for $\mathbb{R}^{d}$ associated with the lattice $\mathbb{Z}^{d}$. Additionally, since $\varphi$ is a measurepreserving map, $m\left(\varphi\left([0,1)^{d}\right)\right)=1$ and this shows that $\left.\varphi\left([0,1)^{d}\right)\right)$ tiles $\mathbb{R}^{d}$ by $\mathbb{Z}^{d}$.

6.2. Necessary conditions for $d=2$. In the subsequent subsection, we will prove that for the special case where $d=2$, Theorem 6.1 is the best result that can be obtained under the restriction that the Jacobian of $\varphi$ is upper-triangular, $\varphi$ is invertible, $\varphi\left([0,1)^{2}\right)$ tiles $\mathbb{R}^{2}$ by $\mathbb{Z}^{2}$ and satisfies some additional technical restrictions which we shall clarify.

Theorem 6.4. Let $\varphi: \mathbb{R}^{2} \rightarrow \mathbb{R}^{2}$ such that $\varphi\left(x_{1}, x_{2}\right)=\left(\varphi_{1}\left(x_{1}, x_{2}\right), \varphi_{2}\left(x_{1}, x_{2}\right)\right)$ for some bivariate, real-valued functions $\varphi_{1}$ and $\varphi_{2}$. Assuming additionally that (a) all second-order mixed partial derivatives of $\varphi_{1}, \varphi_{2}$ are continuous (b) $J_{\varphi}\left(x_{1}, x_{2}\right)$ is an upper-triangular matrix, (c) $\operatorname{det} J_{\varphi}\left(x_{1}, x_{2}\right)=1$, then

$$
\varphi\left(x_{1}, x_{2}\right)=\left(z\left(x_{2}\right) x_{1}+f\left(x_{2}\right), \int_{1}^{x_{2}} \frac{1}{z(t)} d t+K\right)
$$

for some constant $K$ and some functions $z, f \in C^{1}(\mathbb{R})$. Moreover, if $z$ is taken to be the constant function 1 , then $\varphi$ is necessarily as described in Theorem 6.1 and $E\left(\mathbb{Z}^{d}, \varphi\right)$ is an orthonormal basis for $L^{2}\left([0,1]^{2}\right)$.

Remark 6.5. The assumption that $z$ is equal to the constant function 1 cannot generally be removed in Proposition 6.4 without affecting its conclusion. For instance if $z\left(x_{2}\right)=e^{x_{2}}$ and $f\left(x_{2}\right)=0$ then

$$
\varphi\left(x_{1}, x_{2}\right)=\left(e^{x_{2}} x_{1}, \sinh \left(x_{2}\right)-\cosh \left(x_{2}\right)+K\right) .
$$

for some constant $K$. Next, the Jacobian of $\varphi$ has for determinant

$$
\operatorname{det}\left[\begin{array}{cc}
e^{x_{2}} & x_{1} e^{x_{2}} \\
0 & \cosh \left(x_{2}\right)-\sinh \left(x_{2}\right)
\end{array}\right]_{14}=\left(\cosh x_{2}\right) e^{x_{2}}-\left(\sinh x_{2}\right) e^{x_{2}}=1 .
$$


Therefore, $\varphi$ is Lebesgue-measure preserving. However, it is easy to verify that the collection $E\left(\mathbb{Z}^{d}, \varphi\right)$ is not an orthonormal basis for $L^{2}\left([0,1)^{2}\right)$ since the set $\varphi\left([0,1)^{2}\right) \cap$ $\left(\varphi\left([0,1)^{2}\right)+(1,0)\right)$ has a positive Lebesgue measure in $\mathbb{R}^{2}$.

Let $\varphi: \mathbb{R}^{2} \rightarrow \mathbb{R}^{2}$ such that $\varphi\left(x_{1}, x_{2}\right)=\left(\varphi_{1}\left(x_{1}, x_{2}\right), \varphi_{2}\left(x_{1}, x_{2}\right)\right)$ for some bivariate, real-valued functions $\varphi_{1}$ and $\varphi_{2}$. Assuming additionally that all partial derivatives of $\varphi_{1}$ and $\varphi_{2}$ are defined, and

$$
|\operatorname{det} J \varphi|=\left|\frac{\partial \varphi_{1}}{\partial x_{1}} \cdot \frac{\partial \varphi_{2}}{\partial x_{2}}-\frac{\partial \varphi_{2}}{\partial x_{1}} \cdot \frac{\partial \varphi_{1}}{\partial x_{2}}\right|=1
$$

there does not seem to be a simple way to explicitly described all such functions. However, we will prove that under the additional assumptions that $\varphi\left(x_{1}, x_{2}\right)=\left(\varphi_{1}\left(x_{1}, x_{2}\right), \varphi_{2}\left(x_{2}\right)\right)$ and all second order mixed partial derivatives are continuous, $\varphi$ can be described quite explicitly as stated in the lemma below

Lemma 6.6. Let $\varphi: \mathbb{R}^{2} \rightarrow \mathbb{R}^{2}$ such that $\varphi\left(x_{1}, x_{2}\right)=\left(\varphi_{1}\left(x_{1}, x_{2}\right), \varphi_{2}\left(x_{2}\right)\right)$ for some bivariate, real-valued functions $\varphi_{1}$ and $\varphi_{2}$. Assuming additionally that all second-order mixed partial derivatives of $\varphi_{1}, \varphi_{2}$ are continuous then the following are equivalent.

(1) $J \varphi\left(x_{1}, x_{2}\right)$ is an upper-triangular matrix and $\operatorname{det}\left(J \varphi\left(x_{1}, x_{2}\right)\right)=1$ for all $\left(x_{1}, x_{2}\right) \in$ $\mathbb{R}^{2}$.

(2) There exist differentiable functions $z, f \in C^{1}(\mathbb{R}), z \neq 0$ and some constant $K$ such that $\varphi\left(x_{1}, x_{2}\right)=\left(z\left(x_{2}\right) x_{1}+f\left(x_{2}\right), \int_{1}^{x_{2}} \frac{1}{z(\tau)} d \tau+K\right)$.

Proof. To prove that (2) implies (1), we verify that the Jacobian of the map $\varphi\left(x_{1}, x_{2}\right)=$ $\left(z\left(x_{2}\right) x_{1}+f\left(x_{2}\right), \int_{1}^{x_{2}} \frac{1}{z(\tau)} d \tau+K\right)$ is given by

$$
J \varphi\left(x_{1}, x_{2}\right)=\left[\begin{array}{cc}
z\left(x_{2}\right) & f^{\prime}\left(x_{2}\right)+x_{1} z^{\prime}\left(x_{2}\right) \\
0 & \frac{1}{z\left(x_{2}\right)}
\end{array}\right] .
$$

For the converse, assume that $J \varphi\left(x_{1}, x_{2}\right)$ is an upper-triangular matrix and $\operatorname{det}\left(J \varphi\left(x_{1}, x_{2}\right)\right)=$ 1 for all $\left(x_{1}, x_{2}\right) \in \mathbb{R}^{2}$. In other words,

$$
J \varphi(x, y)=\left[\begin{array}{ll}
\frac{\partial \varphi_{1}\left(x_{1}, x_{2}\right)}{\partial x_{1}} & \frac{\partial \varphi_{1}\left(x_{1}, x_{2}\right)}{\partial x_{2}} \\
\frac{\partial \varphi_{2}\left(x_{1}, x_{2}\right)}{\partial x_{1}} & \frac{\partial \varphi_{2}\left(x_{1}, x_{2}\right)}{\partial x_{2}}
\end{array}\right]=\left[\begin{array}{cc}
\frac{\partial \varphi_{1}\left(x_{1}, x_{2}\right)}{\partial x_{1}} & \frac{\partial \varphi_{1}\left(x_{1}, x_{2}\right)}{\partial x_{2}} \\
0 & {\left[\frac{\partial \varphi_{1}\left(x_{1}, x_{2}\right)}{\partial x_{1}}\right]^{-1}}
\end{array}\right]
$$

By Clairaut's theorem, since all second-order mixed partial derivatives of $\varphi_{1}, \varphi_{2}$ are continuous,

$$
0=\frac{\partial}{\partial x_{2}}\left(\frac{\partial \varphi_{2}\left(x_{1}, x_{2}\right)}{\partial x_{1}}\right)=\frac{\partial}{\partial x_{1}}\left(\frac{\partial \varphi_{2}\left(x_{1}, x_{2}\right)}{\partial x_{2}}\right)=\frac{\partial}{\partial x_{1}}\left(\left[\frac{\partial \varphi_{1}\left(x_{1}, x_{2}\right)}{\partial x_{1}}\right]^{-1}\right)
$$

and

$$
-\left[\frac{\partial \varphi_{1}\left(x_{1}, x_{2}\right)}{\partial x_{1}}\right]^{-2} \cdot\left(\frac{\partial^{2} \varphi_{1}\left(x_{1}, x_{2}\right)}{\partial x_{1}^{2}}\right)=0
$$


As a result, $\frac{\partial^{2} \varphi_{1}\left(x_{1}, x_{2}\right)}{\partial x_{1}^{2}}=0$ and this holds if and only if $\varphi_{1}\left(x_{1}, x_{2}\right)=f\left(x_{2}\right)+x_{1} z\left(x_{2}\right)$ for some $z, f \in C^{1}(\mathbb{R}), z \neq 0$. On the other hand,

$$
\frac{\partial \varphi_{2}\left(x_{1}, x_{2}\right)}{\partial x_{2}}=\left[\frac{\partial \varphi_{1}\left(x_{1}, x_{2}\right)}{\partial x_{1}}\right]^{-1} \Leftrightarrow \varphi_{2}\left(x_{1}, x_{2}\right)=\int_{1}^{x_{2}} \frac{1}{z(\tau)} d \tau+K\left(x_{1}\right) .
$$

Finally, since

$$
\frac{\partial}{\partial x_{1}}\left(\varphi_{2}\left(x_{1}, x_{2}\right)\right)=\frac{\partial}{\partial x_{1}}\left(\int_{1}^{x_{2}} \frac{1}{z(\tau)} d \tau+K\left(x_{1}\right)\right)=K^{\prime}\left(x_{1}\right)=0
$$

$K$ must be a constant quantity.

Proof of Theorem 6.4. The first part of the theorem is proved in Lemma 6.6. Assume that $z\left(x_{2}\right)=1$ for all $x_{2} \in \mathbb{R}$. Then

$$
\varphi\left(x_{1}, x_{2}\right)=\left(x_{1}+f\left(x_{2}\right), \int_{1}^{x_{2}} d t+K\right)=\left(x_{1}+f\left(x_{2}\right), x_{2}-1+K\right)
$$

for some constant $K$ and Theorem 6.4 is a direct consequence of Theorem 6.1,

We remark that the converse of Theorem 6.4 is generally false, as shown below.

Proposition 6.7. Under the assumption stated in Theorem 6.4 if $z\left(x_{2}\right)>1$ on some subset of positive measure in $(0,1)$ then $\varphi\left([0,1)^{2}\right)$ does not tile $\mathbb{R}^{2}$ by $\mathbb{Z}^{2}$.

Proof. Suppose that $z>1$ on some subset of positive measure in $(0,1)$. Letting $\psi(t, \xi)=$ $z(t) \xi$ be a function defined on $(0,1) \times(-1,1)$, the range of $\psi$ is given by the set $\bigcup_{t \in(0,1)}(z(t)(-1,1))$ and the set $\{z(t)(-1,1): t \in(0,1)\} \cap \mathbb{Z} \backslash\{0\}$ is not empty. Next, observe that for points $\left(x_{1}, x_{2}\right),\left(y_{1}, x_{2}\right)$ contained in the open set $(0,1)^{2}$, we have

$$
\varphi\left(x_{1}, x_{2}\right)=\left(z\left(x_{2}\right) x_{1}+f\left(x_{2}\right), \int_{1}^{x_{2}} \frac{1}{z(t)} d t+K\right)
$$

and

$$
\varphi\left(y_{1}, x_{2}\right)=\left(z\left(x_{2}\right) y_{1}+f\left(x_{2}\right), \int_{1}^{x_{2}} \frac{1}{z(t)} d t+K\right) .
$$

Taking the difference of the points $\varphi\left(x_{1}, x_{2}\right)$ and $\varphi\left(y_{1}, x_{2}\right)$, gives

$$
\varphi\left(y_{1}, x_{2}\right)-\left(p\left(x_{1}, x_{2}\right)\right)=\left(z\left(x_{2}\right) y_{1}-z\left(x_{2}\right) x_{1}, 0\right)=\left(z\left(x_{2}\right)\left(y_{1}-x_{1}\right), 0\right) .
$$

Since $y_{1}-x_{1} \in(-1,1)$, we may select $x_{2} \in(0,1)$ such that $z\left(x_{2}\right)\left(y_{1}-x_{1}\right) \in \mathbb{Z} \backslash\{0\}$. This shows that there exists a nonzero element $k \in \mathbb{Z}^{2}$ such that the Lebesgue measure of the set $\left(\varphi(0,1)^{2}+k\right) \cap \varphi\left((0,1)^{2}\right)$ is strictly positive. This means that $\varphi\left([0,1)^{2}\right)$ does not tile $\mathbb{R}^{2}$ by $\mathbb{Z}^{2}$.

\section{Applications to the discretization problem of Representations of LOCALLY COMPACT GROUPS}

In this section, we provide some additional motivation for our work by making a connection between Question 1 and the discretization problem of representations of locally compact groups for the construction of frames and orthogonal bases $[11,12,22,23,21,17$.

For a large class of (solvable Lie) groups, the explicit realization of the action of an infinite-dimensional representation is commonly described in terms of a system involving exponential functions with phases which are generally non-linear. Since these constructions 
may not be readily accessible in the literature to non-specialists, we shall present some examples to motivate the results contained in this section and we will connect them with well-studied systems such as wavelets, Gabor wavelets and shearlets.

Example 7.1. (Gabor orthonormal bases and the Heisenberg group) Let $G=\mathbb{R}^{2} \rtimes \mathbb{R}$ be a semi-direct product group with multiplication given by

$$
(v, t)(w, s)=\left(w+\left[\begin{array}{cc}
1 & t_{1} \\
0 & 1
\end{array}\right] w, t+s\right) .
$$

$G$ is the three-dimensional Heisenberg group. It is a non-commutative simply connected nilpotent (solvable) Lie group. Next, let $\pi$ be a function taking $G$ into the group of unitary operators acting in $L^{2}(\mathbb{R})$ as follows:

$$
[\pi(v, s) f](t)=e^{2 \pi i(1,-t) \cdot\left(v_{1}, v_{2}\right)} f(t-s) \quad\left(f \in L^{2}(\mathbb{R})\right) .
$$

It is not hard to verify that $\pi$ is a continuous group homomorphism. In fact, $\pi$ is an irreducible representation of the Heisenberg group known as a Schrödinger representation. Since $\mathcal{E}=\left\{\mathcal{E}_{k}: x \mapsto e^{2 \pi i x k} \chi_{[0,1)}(x): k \in \mathbb{Z}\right\}$ is an orthonormal basis for $L^{2}[0,1)$ and $\{[0,1)+k: k \in \mathbb{Z}\}$ is a tiling of $\mathbb{R}$, the collection of vectors $\left\{e^{2 \pi i t k} \chi_{[0,1)}(t-l): k, l \in \mathbb{Z}\right\}$ forms an orthonormal basis for $L^{2}(\mathbb{R})$. Note that this basis is obtained from a discrete sampling of the orbit of the indicator function $\chi_{[0,1)}$ under the action of the representation $\pi$. In other words, it is possible to discretize $\pi$ to construct an orthonormal basis for $L^{2}(\mathbb{R})$. This is a standard example that is commonly encountered in time-frequency analysis [11.

The following example suggests that Example 7.1, as discussed above, is just a mere occurrence of a much more general phenomenon.

Example 7.2. (Generalized Gabor wavelets) Let $G=\mathbb{R}^{3} \rtimes \mathbb{R}^{2}$ be a semi-direct product group with multiplication given by

$$
(v, t)(w, s)=\left(w+\left[\begin{array}{ccc}
1 & t_{1} & \frac{t_{1}^{2}}{2}+t_{2} \\
0 & 1 & t_{1} \\
0 & 0 & 1
\end{array}\right] w, t+s\right)
$$

Note that although $G$ and $\mathbb{R}^{3} \times \mathbb{R}^{2}$ share the same topological structure, their group structures are quite different. More precisely, $G$ is a non-commutative Lie group [3]; and similarly to the Heisenberg group, its irreducible representations can be exploited to construct an orthonormal basis for $L^{2}\left(\mathbb{R}^{2}\right)$ [21, Example 31]. To see this, let $p: \mathbb{R}^{2} \rightarrow \mathbb{R}^{3}$ be a vector-valued polynomial map defined as follows: $p\left(t_{1}, t_{2}\right)=\left(1,-t_{1},-t_{2}+\frac{t_{1}^{2}}{2}\right)$. Note that the third coordinate of $p$ is a bivariate non-linear polynomial. Next, the function $\pi$ mapping the group $G$ into the group of unitary operators acting in $L^{2}\left(\mathbb{R}^{2}\right)$ as follows

$$
[\pi(v, s) f](t)=e^{2 \pi i p\left(t_{1}, t_{2}\right) \cdot v} f(t-s) \quad\left(f \in L^{2}\left(\mathbb{R}^{2}\right)\right)
$$

can be shown to be an irreducible representation (a continuous homomorphism) of the group $G$. Moreover, it is easy to verify that the system of exponentials with non-linear phase

$$
\left\{\left(t_{1}, t_{2}\right) \mapsto e^{2 \pi i p\left(t_{1}, t_{2}\right) \cdot\left(0, k_{1}, k_{2}\right)} \chi_{[0,1)^{2}}\left(t_{1}, t_{2}\right):\left(k_{1}, k_{2}\right) \in \mathbb{Z}^{2}\right\}
$$

is an orthonormal basis for $L^{2}\left([0,1)^{2}\right)$. This observation together with the fact that $\left\{[0,1)^{2}+k:\left(k_{1}, k_{2}\right) \in \mathbb{Z}^{2}\right\}$ is a measurable partition of $\mathbb{R}^{2}$ imply that the collection of 
vectors

$$
\left\{\left(t_{1}, t_{2}\right) \mapsto e^{2 \pi i\left(-\left(t_{1}+\ell_{1}\right),-\left(t_{2}+\ell_{2}\right)+\frac{\left(t_{1}+\ell_{1}\right)^{2}}{2}\right) \cdot\left(k_{1}, k_{2}\right)} \chi_{[0,1)^{2}}\left(t_{1}+\ell_{1}, t_{2}+\ell_{2}\right):\left(k_{1}, k_{2}, \ell_{1}, \ell_{2}\right) \in \mathbb{Z}^{4}\right\}
$$

is an orthonormal basis for $L^{2}\left(\mathbb{R}^{2}\right)$. In other words, a suitable discretization of the representation $\pi$ gives an orthonormal basis for $L^{2}\left(\mathbb{R}^{2}\right)$.

Example 7.3. (The ax+b group) Let $G=\mathbb{R} \rtimes \mathbb{R}$ be a semidirect product group equipped with the group operation $(x, t)(y, s)=\left(y+e^{t} y, t+s\right)$. Then $G$ is isomorphic to the $\mathrm{ax}+\mathrm{b}$ Lie group which is known to be the group theoretical foundation of wavelet theory [2]. Given a fixed positive real number $\ell$ the unitary representation $\pi_{\ell}$ of $G$ acting in $L^{2}(\mathbb{R})$ as follows:

$$
\pi_{\ell}(x, t) f(s)=e^{2 \pi i e^{-s} \ell x} f(s-t), \quad\left(f \in L^{2}(\mathbb{R})\right)
$$

is irreducible. Put $\varphi(s)=e^{-s} \ell$ and let $L$ be the Lebesgue measure on the real line. Since $\varphi$ is injective, and since the pushforward of the Lebesgue measure via $\varphi$ is a weighted Lebesgue measure of the form $\varphi_{*} L=\frac{1}{x} d x$ on $(0, \infty)$, we obtain the following. Given any positive real number $\epsilon$ and for a fixed countable set $\Lambda \subset \mathbb{R}$ such that the lower Beurling density of $\Lambda$ is positive, and $\left\{e^{2 \pi i \lambda \cdot x}\right\}_{\lambda \in \Lambda}$ forms a Fourier frame for $L^{2}\left(\left[e^{-\epsilon}, e^{\epsilon}\right)\right)$, the system $\left\{\pi_{\ell}(\lambda, 0) 1_{[-\epsilon, \epsilon)}: \lambda \in \Lambda\right\}$ is a Fourier frame for $L^{2}([-\epsilon, \epsilon))$ (see Theorem 3.5.) Next, since $\{[-\epsilon, \epsilon)+\kappa: \kappa \in 2 \epsilon \mathbb{Z}\}$ tiles the real line, it follows that

$$
\left\{\pi_{\ell}\left(e^{\kappa} \lambda, \kappa\right) 1_{[-\epsilon, \epsilon)}: \lambda \in \Lambda \text { and } \kappa \in 2 \epsilon \mathbb{Z}\right\}
$$

is a frame for $L^{2}(\mathbb{R})$.

Example 7.4. (A shearlet group, [22, 21]) Let $G=\mathbb{R}^{2} \rtimes \mathbb{R}^{2}$ be a semi-direct product group with multiplication given by

$$
(v, t)(w, s)=\left(w+\left[\begin{array}{cc}
e^{t_{1}} & t_{2} e^{t_{1}} \\
0 & e^{t_{1}}
\end{array}\right] w, t+s\right) .
$$

Let $\varphi: \mathbb{R}^{2} \rightarrow \varphi\left(\mathbb{R}^{2}\right)$ be a vector-valued smooth map defined as follows: $\varphi\left(t_{1}, t_{2}\right)=$ $\left(e^{-t_{1}},-t_{2} e^{-t_{1}}\right)$. Define a unitary representation $\pi$ of $G$ acting in $L^{2}\left(\mathbb{R}^{2}\right)$ as follows:

$$
[\pi(v, s) f](t)=e^{2 \pi i \varphi\left(t_{1}, t_{2}\right) \cdot v} f(t-s) \quad\left(f \in L^{2}\left(\mathbb{R}^{2}\right)\right) .
$$

Then it is not difficult to verify that $\pi$ is an irreducible representation of $G$. Note also that $\varphi$ is injective. In fact, $\varphi$ defines a diffeomorphism between its domain and its range. Next, let $\Lambda$ be a countable subset of $\mathbb{R}^{2}$ such that the pushforward of Lebesgue measure on $[0,1)^{2}$ to $\varphi\left([0,1)^{2}\right)$ is a frame-spectral measure with frame spectrum $\Lambda$. Then the system

$$
\left\{t \mapsto e^{2 \pi i \varphi(t+l) \cdot k} \chi_{[0,1)^{2}}(t+l):(k, l) \in \Lambda \times \mathbb{Z}^{2}\right\}
$$

is a frame for $L^{2}\left(\mathbb{R}^{2}\right)$.

To generalize the examples above, we will appeal to the results in Theorem 3.2 to derive some sufficient conditions under which a class of unitary representations of some connected Lie groups [12, 22, 21 can be discretized, for the construction of orthogonal bases and frames in $L^{2}\left(\mathbb{R}^{m}\right)$. 
To this end, let $G=\mathbb{R}^{d} \rtimes \mathbb{R}^{m}$ be a connected semidirect product group endowed with the following binary operation: $(x, t)(y, s)=(x+t \bullet y, t+s),(x, t),(y, s) \in G$ where

$$
t \bullet y=\exp \left(\sum_{k=1}^{m} t_{k} A_{k}\right) y
$$

and $A_{1}, \cdots, A_{m}$ is a sequence of pairwise commuting square matrices of order $d$.

Let $\pi$ be a representation of $G$ acting unitarily in $L^{2}\left(\mathbb{R}^{m}\right)$ as follows. Given $f \in$ $L^{2}\left(\mathbb{R}^{m}\right),(x, t) \in G$ and a fixed vector $\ell \in \mathbb{R}^{d}$, we define

$$
\pi(x, t) f(s)=e^{2 \pi i(\varphi(s) \cdot x)} f(s-t)
$$

where the mapping $\varphi: \mathbb{R}^{m} \longrightarrow \mathbb{R}^{d}$ given by $\varphi(t)=\exp \left(-\sum_{k=1}^{m} t_{k} A_{k}\right)^{T} \ell$ is a smooth function. Note that for each $x \in \mathbb{R}^{d}$, the operator $\pi(x, e)$ acts by multiplication with an exponential function with a nonlinear phase (generally) and the conormal part of $G$ acts by translations. In light of these observations, the action of $G$ in $L^{2}\left(\mathbb{R}^{m}\right)$ can be viewed as a form of generalized time-frequency shift.

A straightforward application of Theorem 3.2, gives the following.

Proposition 7.5. If there exist a countable set $\Lambda \subset \mathbb{R}^{d}$ and $\Omega, \Gamma \subset \mathbb{R}^{m}$ such that (a) $\varphi$ is $\mu$-essentially injective, (b) the pushforward of the Lebesgue measure on $\mathbb{R}^{m}$ restricted to $\Omega$ is a (frame) spectral measure with (frame) spectrum $\Lambda$, (c) $\{\Omega+\gamma: \gamma \in \Gamma\}$ tiles $\mathbb{R}^{m}$, then the system

$$
\left\{s \mapsto e^{2 \pi i \varphi(s-\gamma) \cdot \lambda} 1_{\Omega}(s-\gamma): \lambda \in \Lambda, \gamma \in \Gamma\right\}
$$

is an orthogonal basis (or a frame) for $L^{2}\left(\mathbb{R}^{m}\right)$. In other words,

$$
\left\{\pi(\gamma \cdot \lambda, \gamma) 1_{\Omega}:(\lambda, \gamma) \in \Lambda \times \Gamma\right\}
$$

is an orthogonal basis (or a frame) for $L^{2}\left(\mathbb{R}^{m}\right)$.

Proof. By assumption, the system $\left\{s \mapsto e^{2 \pi i \varphi(s) \cdot \lambda} 1_{\Omega}(s): \lambda \in \Lambda\right\}$ is an orthogonal basis (or a frame) for $L^{2}(\Omega)$. Moreover, since each $\pi(\gamma), \gamma \in \Gamma$ is a unitary operator and since the image of an orthogonal basis (or a frame) under a unitary map is an orthogonal basis (or a frame), it follows that for a fixed $\gamma \in \Gamma$,

$$
\pi(0, \gamma)\left(\left\{s \mapsto e^{2 \pi i \varphi(s) \cdot \lambda} 1_{\Omega}(s): \lambda \in \Lambda\right\}\right)
$$

is an orthogonal basis (or a frame) for $L^{2}(\Omega+\gamma)$. Finally, since $\{\Omega+\gamma: \gamma \in \Gamma\}$ is a measurable partition of $\mathbb{R}^{m}$, it follows that

$$
\begin{aligned}
& \bigcup_{\gamma \in \Gamma} \pi(0, \gamma)\left(\left\{s \mapsto e^{2 \pi i \varphi(s) \cdot \lambda} 1_{\Omega}(s): \lambda \in \Lambda\right\}\right) \\
& =\left\{s \mapsto e^{2 \pi i \varphi(s-\gamma) \cdot \lambda} 1_{\Omega}(s-\gamma): \lambda \in \Lambda, \gamma \in \Gamma\right\}
\end{aligned}
$$

is an orthogonal basis (or a frame) for $L^{2}\left(\mathbb{R}^{m}\right)$.

\section{Open problems}

This paper provides a systematic study about the generalized exponential system $E(\Lambda, \varphi)$ forming a frame and basis in some $L^{2}(\mu)$. We are left with many questions that we have not been able to provide a complete answer. 
(1) Given any finite Borel measure $\mu$ on $\mathbb{R}^{d}$. Due to the flexibility of Borel measurable functions $\varphi$, is it true that every $L^{2}(\mu)$ can admit some $E(\Lambda, \varphi)$ as an orthogonal basis? Can we find a Borel measure that does not admit any orthogonal basis of non-linear phase?

(2) The middle-third Cantor measures example given in Section 4 admits orthogonal basis of exponentials with a non-linear phase function $\varphi$. They are not differentiable everywhere and is drastically different from our familiar phase functions $\varphi(x)=x$. A natural question here is that can we find $\varphi$, defined in an open set containing the support, of better regularity (e.g., $\varphi$ is $C^{\infty}$ ) so that $E(\Lambda, \varphi)$ forms an orthogonal basis? For other open, connected sets, do we have an exponential orthogonal basis with a non-linear phase?

(3) Theorem 5.1 provides a characterization on $\mathbb{R}^{1}$ that a continuous function can be an orthogonal basis for $L^{2}[0,1]$ with integer frequencies, provided that $\varphi$ preserves measure zero sets. It looks like that there may exist a continuous function, which will be highly irregular, such that $E(\mathbb{Z}, \varphi)$ forms a basis or a frame for $L^{2}[0,1]$. Will there be any such function? Or can we remove the preserving measure-zero set assumption in Theorem 5.1]

(4) Theorem 6.1 provides a large class of functions that $E\left(\mathbb{Z}^{d}, \varphi\right)$ can form an orthogonal basis for $L^{2}[0,1]^{d}$. Are there any other $C^{1}$-functions $\varphi$ not of the form

$$
x \mapsto M\left(x_{1}+l_{1}\left(x_{2}, \cdots x_{d}\right), x_{2}+l_{2}\left(x_{3}, \cdots, x_{d}\right), \cdots, x_{d-1}+l_{d-1}\left(x_{d}\right), x_{d}\right)
$$

for some $C^{1}$-functions $l_{1}, l_{2}, \cdots, l_{d-1}$ where $M$ is a matrix in integer entries satisfying $|\operatorname{det} M|=1$ for which $E\left(\mathbb{Z}^{d}, \varphi\right)$ is an orthonormal basis for $L^{2}[0,1]^{d}$ ?

\section{REFERENCES}

[1] David Bernier and Keith F. Taylor. Wavelets from square-integrable representations. SIAM J. Math. Anal., 27(2):594-608, 1996.

[2] Ole Christensen. An introduction to frames and Riesz bases, volume 7. Springer, 2003.

[3] Laurence Corwin and Frederick P Greenleaf. Representations of Nilpotent Lie Groups and Their Applications: Volume 1, Part 1, Basic Theory and Examples, volume 18. Cambridge university press, 1990.

[4] R. J. Duffin and A. C. Schaeffer. A class of nonharmonic Fourier series. Trans. Amer. Math. Soc., 72:341-366, 1952.

[5] Dorin Ervin Dutkay and Chun-Kit Lai. Uniformity of measures with fourier frames. Advances in Mathematics, 252:684-707, 2014.

[6] Dorin Ervin Dutkay, Chun-Kit Lai, and Yang Wang. Fourier bases and Fourier frames on selfaffine measures. In Recent developments in fractals and related fields, Trends Math., pages 87-111. Birkhäuser/Springer, Cham, 2017.

[7] Hans G. Feichtinger and Peter Gröbner. Banach spaces of distributions defined by decomposition methods. I. Math. Nachr., 123:97-120, 1985.

[8] G. J. Foschini. Almost everywhere one-to-one functions and an $n$-cube decomposition. J. Math. Anal. Appl., 31:314-317, 1970.

[9] Bent Fuglede. Commuting self-adjoint partial differential operators and a group theoretic problem. $J$. Functional Analysis, 16:101-121, 1974.

[10] Jean-Pierre Gabardo, Chun-Kit Lai, and Yang Wang. Gabor orthonormal bases generated by the unit cubes. J. Funct. Anal., 269(5):1515-1538, 2015.

[11] Karlheinz Gröchenig. Foundations of time-frequency analysis. Applied and Numerical Harmonic Analysis. Birkhäuser Boston, Inc., Boston, MA, 2001.

[12] Karlheinz Gröchenig and David Rottensteiner. Orthonormal bases in the orbit of square-integrable representations of nilpotent lie groups. arXiv preprint arXiv:1706.06034, 2017. 
[13] Xing-Gang He, Chun-Kit Lai, and Ka-Sing Lau. Exponential spectra in $l^{2}(\mu)$. Applied and Computational Harmonic Analysis, 34(3):327-338, 2013.

[14] Christopher Heil. A basis theory primer: expanded edition. Springer Science \& Business Media, 2010.

[15] Adrian Holhos. Two area preserving maps from the square to the p-ball. Math. Model. Anal., 22(2):157-166, 2017.

[16] Palle ET Jorgensen and Steen Pedersen. Dense analytic subspaces in fractall 2-spaces. Journal dAnalyse Mathematique, 75(1):185-228, 1998.

[17] Alexander S. Kechris. Classical descriptive set theory, volume 156 of Graduate Texts in Mathematics. Springer-Verlag, New York, 1995.

[18] Gady Kozma and Shahaf Nitzan. Combining Riesz bases. Invent. Math., 199(1):267-285, 2015.

[19] Gady Kozma and Shahaf Nitzan. Combining Riesz bases in $\mathbb{R}^{d}$. Rev. Mat. Iberoam., 32(4):1393-1406, 2016.

[20] Nir Lev and Mate Matolcsi. The fuglede conjecture for convex domains is true in all dimensions. https://arxiv.org/abs/1904.12262.

[21] Vignon Oussa. Compactly supported bounded frames on lie groups. Preprint.

[22] Vignon Oussa. Frames arising from irreducible solvable actions i. Journal of Functional Analysis, 274(4):1202-1254, 2018.

[23] Vignon S Oussa. Regular sampling on metabelian nilpotent lie groups: The multiplicity-free case. In Frames and Other Bases in Abstract and Function Spaces, pages 377-411. Springer, 2017.

[24] Robert S. Strichartz. Mock Fourier series and transforms associated with certain Cantor measures. $J$. Anal. Math., 81:209-238, 2000.

[25] Terence Tao. Fuglede's conjecture is false in 5 and higher dimensions. Math. Res. Lett., 11(2-3):251258,2004

[Jean-Pierre Gabardo] Department of Mathematics, McMaster University, Hamilton, Canada. E-mail address: gabardo@mcmaster.ca

[Chun-Kit Lai] Department of Mathematics, San Francisco State University, San FranCISCO, US.

E-mail address: cklai@sfsu.edu

[Vignon Oussa] Department of Mathematics, Bridgewater State University Massachusetts, US.

E-mail address: vignon.oussa@bridgew.edu 